\title{
Aerodynamics of the Wing/Fuselage Junction at an Transport Aircraft in High-lift Configuration
}

\author{
Stefan Melber-Wilkending (Stefan.Melber@DLR.de) \\ German Aerospace Center (DLR), Institute of Aerodynamics and Flow Technology \\ Lilienthalplatz 7, D-38108 Braunschweig, Germany
}

\begin{abstract}
Summary
This paper presents the numerical simulation (DLR TAU-code) and the analysis of viscous high-lift flow around a complex wing/body configuration (DLR ALVAST) in landing configuration. The investigations aim at a better understanding of the aerodynamics at the wing root and the lift breakdown for such a configuration.
\end{abstract}

\section{Introduction}

The optimization of a transport aircraft at high incidence with respect to low speed take-off/landing capabilities and handling qualities near wing stall is a complex aerodynamic problem. The aerodynamic characteristic of such a high-lift configuration is determined by the type of high-lift devices and its settings. There are in addition some critical areas like the engine/nacelle integration or the wing/fuselage junction, which trigger premature flow separation. A triggered wing stall is usually advantageous for handling quality reasons, but it limitates the maximum lift. The design of the AIRBUS A321 [1] is an example for such optimization aiming on the wing root flow, especially on the inner slat-end/fuselage juncture. It was found, that a small device at the inner slat end delays the wing root stall significantly [2].

The two main tools to study such aerodynamic effects are on one hand windtunnel testing which provide mature measurement techniques and is therefore the basis of the industrial aircraft development. On the other hand, fast low order design methods are used, which however have only a limited accuracy in forecasting three-dimensional effects. Therefore increasingly higher order numerical methods like volume methods based on the Navier-Stokes-equations coming in business [3] which compared to windtunnel experiments holds the promise to significantly accelerate the aerodynamic design, save costs and give a detailed insight into the flow field.

This paper continues the investigations of the DLR ALVAST wing/body transport aircraft configuration in high-lift condition [4], [5]. The focus is mainly on an improved understanding of the complex flow field, which is dominated by effects caused by the three-dimensionality. Moreover, the current reproduction of the stall effects and the lift breakdown as were found in the windtunnel tests depending from the geometry in the wing/body junction is another objective. 


\section{Numerical Method, Geometry and Meshes}

The solution of the Reynolds-averaged Navier-Stokes equations (RANS) is carried out using the hybrid unstructured DLR TAU code [6]. For the closure of the Reynolds-averaged equations the k- $\omega$-SST turbulence model of Menter is used, which combines robustness with the applicability for partly detached flows. Due to the low Mach numbers and the resulting stiffness of the RANS equations, low Mach number preconditioning is used. Further on, the laminar/turbulent transition is detected automatically and accounted in the numerical simulations, which also prevents the so called stagnation point anomaly of the k- $\omega$-SST model. The central JST-scheme in combination with $80 \%$ matrix dissipation assures numerical flow solutions with low numerical dissipation. Further on, the numerical windtunnel is used to remove uncertainties because of half-model testing, windtunnel corrections and angle-of-attack hysteresis [7].

The configuration considered in this paper is the ALVAST transport aircraft geometry, a generic configuration comparable with a modern, two-engine transport aircraft like an AIRBUS A320 (scale 1 : 10). The slat on the landing configuration used here is deployed at $27.0^{\circ}$, the single slotted flap at $32.0^{\circ}$. The half-model was placed on a peniche (height $68 \mathrm{~mm}$ ) in the low-speed windtunnel DNW-NWB (Braunschweig, cross-section $3.25 \mathrm{~m} \times 2.80 \mathrm{~m}$ ). The free-stream conditions are a velocity of $\left|\boldsymbol{V}_{\infty}\right|=60 \mathrm{~m} / \mathrm{s}$ and a Reynolds-number of $R e_{\infty}=1.435 \cdot 10^{6}$ with an reference length $l_{\mu}=0.41 \mathrm{~m}$. The hybrid unstructured meshes for the numerical simulation are generated grid generator Centaur of CentaurSoft [8], for details see [7].

\section{Results}

For the ALVAST-configuration a large number of measurements on national and also European level [9] are available. Concerning the achievable maximum lift and the flow in the wing/body junction the following effects were found: a flow separation in the wing-root section is the limiting factor regarding the maximum lift of the ALVAST-configuration and can be considerable reduced and moved to higher angles of attack by an fairing on the upper-side of the wing and mainly by closing the gap between the inboard slat-end and the fuselage. However on an real aircraft this gap cannot be closed because of the necessary motion of the slat and therefore the effect of an end-plate including a fillet on the inboard slat end (slathorn) was investigated, which shows nearly the same positive effect as closing the gap [9].

A variation of the geometry in the area of the wing/body junction during the windtunnel test shows the following sensitivity Figure 1: while the geometry with slathorn and wing/body fairing (3) and with a small reduction the geometry with slathorn alone (4) reaches the highest maximum lift, both geometries without slathorn and with/without fairing ( 2 and 1 ) has a significantly lower lift, compare Figure 2. In this both cases the same small reduction can be found without a fairing. Summarized it can be found the slathorn has a considerable influence on the achievable maximum lift, whereas the fairing has a supporting effect. 
Further on in this Figure the results of the numerical simulation are shown. Compared with the measurements the influence of the geometry variations in the wing/body junction for configurations 1 -3 are correct reproduced regarding the maximum lift behavior, whereas configuration 4 shows a clear deviation. A detailed investigation regarding the different influences like the turbulence model, the behavior of local flow separations, differences in the model-geometry compared to the CAD-model, the deformation of the windtunnel model and the holders of the slatand flap-segments was carried out. Because of the limited length of the paper this results cannot be shown here.

In the following the differences in the flow fields will be traced back on the variations of the geometry and with it the determination of the mechanism of the maximum lift. In general the lift breakdown does not take place immediate after a topological change in the flow field, but commonly induced by an existing flow separation, which spreads out with increasing angle of attack and leads to a final lift breakdown. Therefore in a first step flow separations on the ALVAST configuration are detected and checked, if there exists a connection with the geometry variation. On the outboard wing starting with an angle of attack of $\alpha=12^{\circ}$ a flow separation occurs on the trailing edge of the wing. The reason is the pressure rise in the direction of the trailing edge, which is increased in this region because of the missing flap there and therewith finally overstressing the boundary layer. An area of recirculating flow can be found above both flaps without any contact to the surface starting with an angle of attack of $\alpha=10^{\circ}$ caused by the pressure rise inside the slat- and wing-wakes in the flow field.

All shown flow separations discussed until now have no dependency with the geometry in the wing/body junction. However the fairing itself has a significant influence: if no fairing is placed already at lower angles of attack a flow separation spreading out to the trailing edge of the wing can be found. One reason is the mainly at high- and low-wing mounting occurring acute angle between wing and fuselage and the aligned channel, which is growing in the direction to the trailing edge in this area. The downwash of the wing and the spanwise flow is not sufficient to fill up the additional volume, after all the fuselage shields the downwash geometrically. Further on because of necessary movement of the flaps the inboard flap not the complete trailing edge of the inboard wing is covered with a flap, which increases the load on the boundary layer additional.

However the behavior found at maximum lift cannot be completely explained with the faring influence. An example are configurations 2 and 3 which have both an fairing, but a complete different maximum lift behavior (Figure 2). Although in case of configuration 2 starting with an angle of attack of $\alpha=16.5^{\circ}$ a flow separation can be found in this area, which does not spread out till an angle of attack $\alpha=19.5^{\circ}$. Therefore there must be another mechanism which influences the maximum lift. To exclude the influence of the fairing, in the following configurations 2 and 3 will be considered, which have both a faring. Comparing the positions of the vortices (Figure 3) significant differences can be found the position and also in the topology 
of the vortices in the area of the wing/body junction. To determine the influence of the vortices on the maximum lift, they should be discussed in more detail.

In the area of the wing/body junction the boundary layer on the fuselage hits the leading edge of the wing respectively the slatstump. A stagnation point occurs there with a significant pressure rise stream-up, which cannot overcome by the boundary layer of the fuselage and therefore it separates from the fuselage surface. This forms the so called horseshoe vortex, which diverted from the free stream around the wing-root (Figure 3 ) and increases its diameter on the upper wing behind the maximum thickness because of the pressure rise. In (Figure 3, colored in red) overall three (primary) horseshoe vortices can be identified in the wing/body junction: the primary slatstump horseshoe vortex in front of the slatstump, which runs along the fuselage and vanishes after a short distance because of its weakness. The slatedgestump vortex forms on the spanwise outboard side of the slatstump and is strictly speaking no horseshoe vortex, because it is initiated by a local flow separation on an edge of the slatstump. Its rotation direction and behavior is however analog to a horseshoe vortex. After the forming on the edge it unites with the primary slatstump horseshoe vortex. The third primary horseshoe vortex forms in front of the wing on the wing leading edge.

Because of the pressure difference from upper- to lower-side the flow is running around the inboard sideedge of the slat and creating a primary slatsideedge vortex on the upper sideedge rather the slathorn, which propagates on the upper wing to the trailing edge. The rotation direction on the upper wing is the same as for the horseshoe vortices. With increasing angle of attack the vortex is going stronger and moves steeper above the wing because of the free-stream flow. Inside the gap between slat and fuselage respectively the slat stump an accelerated flow occurs because of the reduction of the cross section. This jet-like flow moves in spanwise direction due to the pressure field in this area. Because of the interaction with the gapflow on the slat sideedge the secondary slat sideedge vortex with a rotation direction like the primary slatstump horseshoe vortex, runs cross the free-stream with increasing distance to the upper wing and unites with this vortex. In front and above the primary slat sideedge vortex the induced flow leads to a redirection of the flow and additionally the vortex blocks partially the downwash of the wing, which induces finally a counter-rotating vortex. This secondary horseshoe vortex runs along the fuselage parallel to the inducing primary slatstump horseshoe vortex and moves downwards because of its on induced velocity.

On a swept wing creating lift the flow on the lower side is directed outboard because of the trailing vortices. Therefore in the slat-gap spanwise outboards directed flow can be found with a magnitude comparable to the free-stream velocity. Regarding the flow running from the lower side in the slatgap the cove is a type of undercut and therefore the flow separates and forms a slatcove vortex. In the section of the inboard slatend this spanwise flow runs from the lower trailing in this cove, while inside the cove the flow velocity increases because of the suction peak on the wing leading edge. The slatcove vortex is moved upwards because of this flow and leaves 
the slatgap on the upper side of the wing with a rotation direction counter rotating compared to the horseshoe vortices (Figure 3, colored in blue).

Because of the additional volume needed for the described vortices along the fuselage the conclusion suggests itself this vortex-front has an influence because of its volume, its induced velocity and the shading of the downwash above the wing in the area of the wing/body junction and therefore influence the maximum lift of the configuration. A closer look on the cross-flow shows however only in small distances from the vortex-front a significant induced flow can be found and the shading effect of the vortex front plays no significant role.

The differences in the position of the vortices for configuration 2 and 3 can be found already in the area of the slat- and wing-leading edge (Figure 3). A detailed analysis in this area shows in case of the configuration with slathorn (3) the primary slat sideedge vortex leaving the surface earlier and has in spanwise direction a closer distance to the surface of the fuselage. At the same time the rollup of the vortex starts earlier on the slat sideedge, which indicates a stronger vortex compared to the case without the slathorn. The mounting of the slathorn on a sheet of metal with an finite thickness reduces the gap between the slatsideedge and the slatstump and further on because of the increased size of the sideedge the gap has an increased size in flow direction. The increased drag in the gap reduces the flow velocity compared with a configuration without a slathorn, but the gap flow itself is more unified and moved farther towards the upper wing surface because of the smaller and longer channel. This leads to an additional deflection of the unified horseshoe vortices more upwards and more inboards compared to the side edge. Further on a better separation of the unified horseshoe vortices with the equal rotating primary slat sideedge vortex occurs. This separation is increased because of the jet-flow from the gap and the increased size of the slat sideedge and leads in the case with slathorn to an delayed mixing of the primary slat sideedge vortex and the unified horseshoe vortices by an longer rotation around each other (Figure 3).

This interaction of two vortices plays now an important role in the further discussion. The mixing behavior of two vortices rotating in the same direction depends from the strength of each partner: in the case without a slathorn (2) the primary slat sideedge vortex is weaker as the unified horseshoe vortices and this leads to a fast mixing. In the contact area because of the opposite induced flow velocity additional dissipation occurs, which increases the size of the vortex and reduces the flow velocity in the resulting vortex core. In the case with slathorn (3) however both vortices have nearly the same strength and therefore rotating around each other with a bigger distance as in the case before. The increased distance reduces the dissipation on the contact surface significantly and over a much longer distance two separated vortices can be found in the flow field (Figure 3), whereas in the case without slathorn the increasing expansion and deceleration in the vortex core leads to an vortex bursting (Figure 3). In Figure 4 a cut of the velocity magnitude perpendicular through the fuselage-axis is shown for both configurations, which clearly shows the additional dissipation in the case without slathorn (2) because of the reduced flow velocity in the vortex core. At an angle of attack of $\alpha=16^{\circ}$ (Figure 3) this behavior is clarified: 
in the case without slathorn (2) shortly after the mixing the resulting vortex bursts, whereas in the case with slathorn (3) no bursting can be found. Rather in this case a slowly increasing vortex diameter of the secondary horseshoe vortex can be found, which is placed above the slowly mixing united horseshoe vortices and the primary slat sideedge vortex. Thereby the mixing point is moving slowly in the direction of the leading edge with increasing angle of attack, whereas the vortex front moves higher above the wing surface because of the free stream direction.

In Figure 3 the different shifting of the vortex front on the upper wing between configuration 2 and 3 can be clearly seen. The difference is because the vortex front in case of configuration 2 bursts early and has therefore only a small amount of induced flow velocity on the fuselage before bursting, clearly marked by the kink in the vortex path Figure 3. On the other hand in case of configuration 3 the vortex front runs much longer, induces itself a flow component directed upwards on the fuselage by a dominating primary slat sideedge-vortex and united horseshoevortices and moves finally against the downwash of the wing upwards. Further on this behavior keeps the area of the wing/body junction free of any vortices. In case of the configuration without slathorn (2) the vortex front with its increased dissipation because of its earlier mixing and the vortex bursting runs induced by the downwash of the wing through the area of the wing/body junction and leads to an additional weakening of the boundary layer in this area, which finally leads to the earlier lift breakdown compared with configuration (3) with an slathorn.

Configuration 1 , which has no slathorn like configuration 2 the shifting of the vortex front in the edge of the wing/body junction is avoided because of the flow separation there. However this leads to an early lift breakdown because this still existing separation because of the missing fairing limits the maximum angle of attack.

\section{Conclusion}

With a systematic analysis of the complex three-dimensional flow field of a transportaircraft configuration the influence of geometric details in the area of the wing/body junction on the achievable maximum lift was shown using the numerical flow simulation including the numerical windtunnel. For the ALVAST high-lift configuration it was found that in the case without a slathorn the early mixing of the primary slat-side edge vortex with the united horseshoe vortices leads to an increased dissipation and finally to a vortex bursting of the vortex-front. Overall the promotion or degradation of the flow between the wing and fuselage has a significant influence on the stall of such a configuration. The windtunnel measurements in case of the already mentioned example of the AIRBUS A321 coincide with the demonstrated flow topology of the ALVAST configuration [1], [2].

\section{References}

[1] Flaig, A.; Hilbig, R.: High-Lift Design for Large Civil Aircraft. AGARD-CP-515, pp. 31-1-31-12, September 1993. 
[2] Schwetzler, D.: Improvement of Maximum Lift in Flight by Optimization of Vortex Flow. High-Lift and Separation Control Conference Proceedings, Univ. of Bath, United Kingdom, 29.-31. M"arz, pp. 4.1-4.10, 1995.

[3] Reckzeh, D.: Aerodynamic design of the high-lift-wing for a Megaliner aircraft. Aerospace Scienence and Technology 7, Elsevier, pp. 107-119, 2003.

[4] Rudnik, R.; Melber, S.; Ronzheimer, A.; Brodersen, O.: Aspects of 3D RANS Simulations for Transport Aircraft High-Lift Configurations. AIAA paper 2000-4326, 2000.

[5] Melber, S.: 3D RANS Simulations for High-Lift Analysis of Transport Aircraft Configurations. Notes on numerical fluid mechanics, Volume 77, Springer Verlag, 2002.

[6] Kroll, N.; Rossow, C.-C.; Schwamborn, D.; Becker, K.; Heller, G.: MEGAFLOW - A Numerical Flow Simulation Tool for Transport Aircraft Design. 23rd ICAS Congress, Toronto, ICAS 2002, 1.5-10.5, 2002.

[7] Melber-Wilkending, S.; Wichmann, G.: Project ForMEx - A new CFD approach for transposition of Wind Tunnel data towards Flight Conditions. 15th AG STAB/DGLR Symposium Darmstadt, 29.11 - 1.12.2006.

[8] Kallinderis, Y.: Hybrid Grids and Their Applications. Handbook of Grid Generation, CRC Press, Boca Raton / London / New York, pp. 25-1 - 25-18, 1999.

[9] Puffert-Meissner, W.: ALVAST Half-Model Wind Tunnel Investigations and Comparison with Full-Span Model Results. DLR IB 129-96/20, 1996.

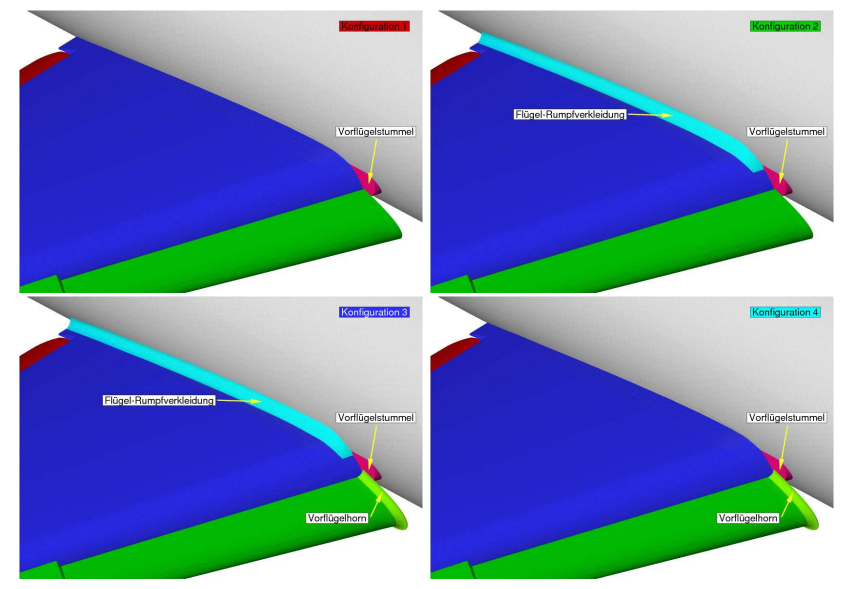

Figure 1 Variation of the geometry of the ALVAST hiftlift configuration in the area of the wing/body junction. Configurations 1-4. 


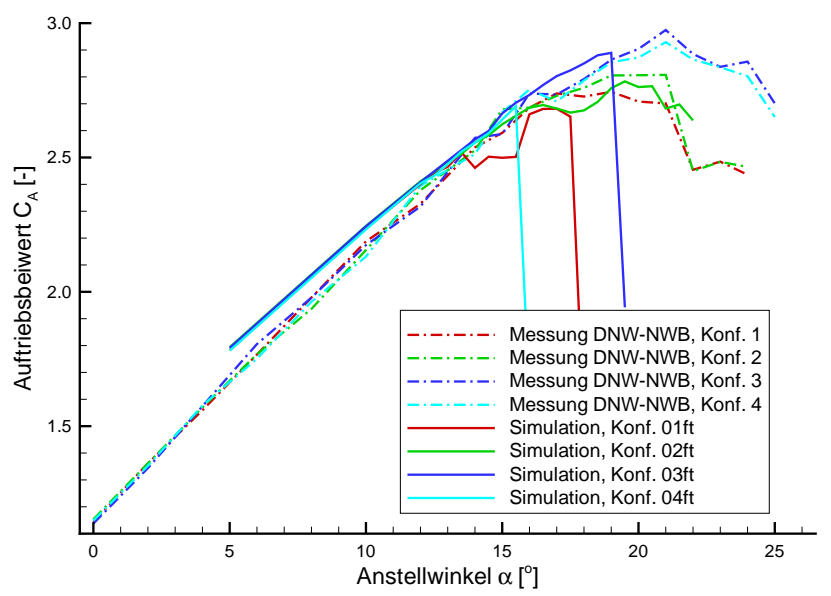

Figure 2 Lift polars of the ALVAST highlift configuration 1-4.

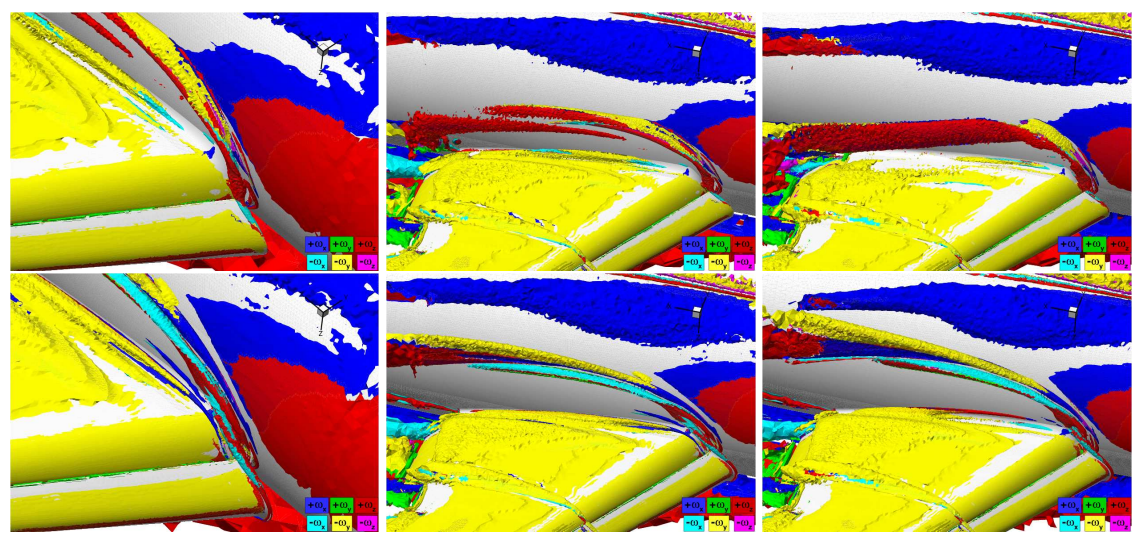

Figure 3 Vortex topology in the area of the wing/body junction, colored with the rotation direction of the vortices, top: configuration 2, bottom 3 , angle of attack $\alpha=14^{\circ}$ (left, middle) and $\alpha=16^{\circ}$ (right).
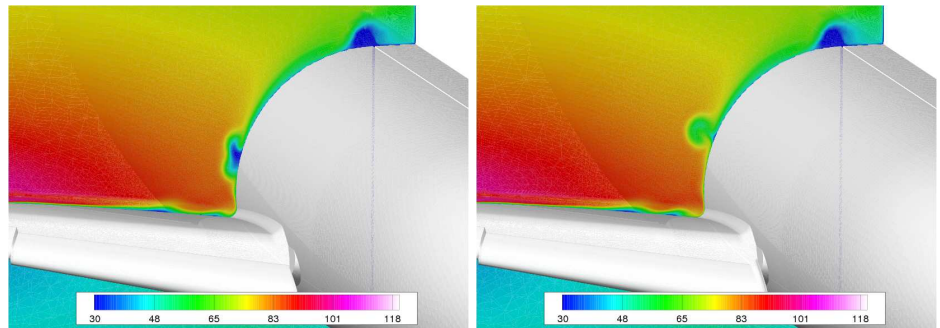

Figure 4 Cut through the flow field perpendicular to the fuselage axis short before the wing trailing edge, magnitude of the velocity $|V|$, Configuration 2 (left) and 3 (right), angle of attack $\alpha=14^{\circ}$. 